\title{
Miranda
}

Revue pluridisciplinaire du monde anglophone /

Multidisciplinary peer-reviewed journal on the English-

speaking world

$12 \mid 2016$

Mapping gender. Old images ; new figures

\section{Queeriosité : le poil a-t-il un genre ? Autour de Del LaGrace Volcano, Daniela Comani, Katarzyna Kozyra, Ana Mendieta et Cindy Sherman}

\section{Frédérique Villemur}

\section{(2) OpenEdition}

Journals

Édition électronique

URL : http://journals.openedition.org/miranda/8732

DOI : 10.4000/miranda.8732

ISSN : 2108-6559

\section{Éditeur}

Université Toulouse - Jean Jaurès

\section{Référence électronique}

Frédérique Villemur, «Queeriosité : le poil a-t-il un genre ? Autour de Del LaGrace Volcano, Daniela Comani, Katarzyna Kozyra, Ana Mendieta et Cindy Sherman », Miranda [En ligne], 12 | 2016, mis en ligne le 02 mars 2016, consulté le 16 février 2021. URL : http://journals.openedition.org/miranda/8732 ; DOI : https://doi.org/10.4000/miranda.8732

Ce document a été généré automatiquement le 16 février 2021.

\section{cc)}

Miranda is licensed under a Creative Commons Attribution-NonCommercial-NoDerivatives 4.0 International License. 


\title{
Queeriosité : le poil a-t-il un genre? Autour de Del LaGrace Volcano, Daniela Comani, Katarzyna Kozyra, Ana Mendieta et Cindy Sherman
}

\author{
Frédérique Villemur
}

De l'inversion des genres au transgenre, le «trans » interroge la notion de frontière et de porosité sans forcément renvoyer au «trans » de la transgression. La peau se signale comme frontière poreuse, le cuir du poil révèlerait à sa manière cet inframince queer à l'identité mouvante (les zones de pilosité faciale et pubienne, mais aussi le reste du corps, supportent des représentations de genres et de sexualités). En confrontant la question esthétique du Nu comme invention d'une catégorie de représentation dans le système des beaux-arts ${ }^{1}$, à celle plus anthropologique de la nudité, quelques observations m'ont conduite à repenser la fonction de marqueur du poil comme indice à la fois d'une animalité et d'une construction culturelle de la différence des genres. Transgresser les canons esthétiques du nu, ou inventer une nouvelle nudité à travers l'inversion des genres, requiert d'arrêter son regard à fleur de peau - la peau, ce qu'il y a de plus profond, pour reprendre ici une formule de Paul Valéry², là où se jouent les identités. La métamorphose des corps dont l'étymologie (meta-morphè) souligne combien il s'agit d'un «changement à travers, au-delà » est à interroger depuis la surface comme limite, alors même que s'y joue une certaine profondeur. On opposera la nudité lisse du nu classique pour lequel le corps féminin est dépourvu de toute pilosité, au corps transgenre où la pilosité migre vers des aires corporelles transitoires, comme au corps glabre d'une esthétique où la nudité semble sublimée au profit d'une confusion des genres. Car «le poil, c'est du corps, mais du corps détachable et modifiable » (Auzépy 5-24). L'approche queer transgresse à la fois des normes sexuées et des codes de genres pour donner lieu à des performances artistiques qui visent tantôt à brouiller les frontières de genres tantôt à les réaffirmer en les déplaçant - rappelons à propos de cette porosité que "queer» a pour racine indo-européenne twerkw qui signifie «à travers $»^{3}$. On distinguera donc dans ces gender-cross performances le 
travestissement (non ce qu'on appelle le cross-dressing, mais l'appropriation d'une nudité exhibant une pilosité acquise par le postiche) du processus transgenre dans lequel le body art est engagé dans une catharsis à la fois esthétique et médicale.

\section{Le déni du poil}

2 Le poil dans tous ses états (le poil facial, les poils pubiens, le poil des dessous de bras, les poils du torse, le poil des jambes) signale le point de surgissement de l'animalité sur notre corps. "Être à poil " c'est être au contact de l'animalité : ainsi en va-t-il de l'expression qui signifie "monter à cru », c'est-à-dire monter sans selle, au contact du dos du cheval. « Être à poil » ou être « tout nu » (1858) est une expression pour laquelle Alain Rey appelle dans le Dictionnaire historique de la langue française (Rey 1992) cet autre synonyme "à cru " ou «monter un cheval à poil » (XVII siècle). Nature bestiale et vitalité naturelle connotent le poil. Le dompter c'est maîtriser l'effroi suscité par l'altérité. La peur du poil c'est avant tout la peur de l'autre ${ }^{4}:$ du satyre au diable hirsute, du dieu Pan au loup-garou, il désigne la sexualité débridée de l'homme-animal. Attaché aux origines les plus secrètes de l'organique, son épilation signe une certaine domestication de la féminité. Du point de vue artistique son absence révèle la ligne lisse du nu académique comme pure enveloppe. Signaler le poil revient à enfreindre les canons du glabre et du lisse dans le Beau, autant que dans l'ordre du quotidien s'affranchir de l'épilation entretient un désir de libération du corps. On a ainsi pu parler de la "revanche des poilues » avec la volonté d'afficher en public un corps en friche dans un univers aseptisé (Vaton 96). Ou récemment, en études de genre, développer ainsi que la presse l'a relaté, des expériences de renversement des genres dans les rapports de sexes, autour de la pilosité 5 . Mais l'exhibition parodique des signes pileux des hommes par le collectif féministe $L a$ Barbe ne les avait-il pas déjà mis en évidence comme insignes d'un pouvoir androcentré ? À chacun donc son poil et la manière de l'arborer à l'endroit visé?

Dans le domaine des beaux-arts, le poil longtemps a été l'enjeu d'un déni systématique dans les genres artistiques canonisés. Rappelons ici quelques traits marquants dans l'histoire de la peinture. Dès l'attribution de l'invention de la peinture à huile par les frères Van Eyck, l'excellence du nouveau médium au regard de sa qualité plastique autant que de la précision qu'il offrait en matière de détail, a incité les peintres à une vérité qui signale pour la première fois la qualité sensuelle de la peau. Les deux panneaux du Retable de l'Agneau mystique ${ }^{6}$, exposant la nudité d'Adam et d'Ève, distinguant la qualité des chairs et la pilosité selon les sexes, finirent par faire scandale $^{7}$, rappelant trop que ces images étaient frappantes et que le poil fait bien la bête... Au $16^{{ }^{2} \mathrm{eme}}$ siècle, avec le premier grand nu pastoral, la Vénus endormie (1507-1510) de Giorgione et Titien ${ }^{8}$, une nouvelle norme se met en place, désignée dans le système des beaux-arts par le terme d'«académie » (une figure nue d'après modèle exprimant une idée du beau par-delà la nudité). Le nu expulse la nudité, bannit toute pilosité à l'endroit du sexe alors même qu'une main bien placée le désigne, ou encore l'endroit des aisselles alors que le geste relevé du bras les découvre de manière érotique. Art de la forme autant que forme d'art, le nu répond à une pulsion scopique qui opère par déplacement ${ }^{9}$. Freud à ce sujet utilise le terme de sublimation: l'objet du voir (les parties génitales) doit pouvoir s'étendre au corps tout entier, être "sublimé » (Freud 1987, 67). La pilosité entre attraction et répulsion, enjeu d'un conflit du désir, devient- 
elle l'objet d'une alternative quant à faire l'homme ou faire la femme? Le travestissement et le renversement des rôles entre les sexes, dans la construction culturelle des genres rapportée à celle des rapports de sexes ne remet pas forcément en question le canon esthétique du nu: ainsi, Rubens dépeignant Hercule et Omphale ${ }^{10}$ dans l'échange de leurs attributs (massue et dépouille de lion, quenouille et robe), rend apparente la virile pilosité du torse et du sexe d'Hercule, tandis qu'il laisse glabre le sexe d'Omphale qu'il recouvre de la queue de la peau de bête. Quant à la mise à nu, qui dénude sans reste ni pudeur, Manet dans l'olympia ${ }^{11}$ (1863) cache par l'à-plat d'une main l'impudique pilosité. Cependant un an auparavant, Gustave Courbet dépeint une femme nue couchée à l'aisselle plus honteusement ombrée ${ }^{12}$ (et ce, bien avant L'Origine $d u$ Monde (1866), qui relève d'une autre commande, et d'une autre facture). Au milieu du siècle encore, on se souvient de la provocation de la Baigneuse endormie près d'une source de Théodore Chassériau ${ }^{13}$ (1850): alors que l'artiste tente de renouveler un thème classique par le recours au naturalisme, une touffe de poils sous les aisselles est pour le public rédhibitoire ${ }^{14}$. La représentation réaliste du corps d'Alice Ozy, l'amante du peintre, détourne le sujet de l'esthétique classique par l'horreur délicieuse d'une chose nouvelle, les poils de l'aisselle, «cette mousse fine que Théophile Gautier a magnifié dans son Musée secret » (Loviot 96-97), et dont la couleur se fond avec celle des frondaisons du paysage. En 1863, la même année que l'Olympia, le public lui préfère la Vénus de Cabanel ${ }^{15}$ pourtant moins discrète dans son étalage de chair au-dessus de l'écume, et elle est dépourvue de duvet et de toison. Révoquer donc la nudité du nu par le déni du poil en un processus d'annulation autant que d'isolation, qui tend à édifier un modèle idéal du corps, revient à confirmer le tabou du toucher, en séparant la forme du désir, ainsi que l'a bien vu Georges Didi-Huberman, concernant le travail de figurabilité (Didi-Huberman 26-31), et le "toucher masqué" évoqué par Freud (Freud 1978, 44-5). Le poil arrête le regard, il retient l'attention, incite à y voir de plus près. Le poil n'est ni une chair, ni une humeur, facilement détachable, voire transposable dans le cas du postiche, imputrescible, qui ne se corrompt jamais, il a partie liée avec la vitalité sauvage de la nudité. Le geste civilisateur lui, tend à polir la peau, ainsi en va-til du glabre comme critère du Beau. Nudité/sexualité vs $\mathrm{Nu}$ /érotisme : c'est en ôtant quelque chose à la nudité que le nu porte présence, ainsi peut-on opposer le comble du nu au dénuement de la nudité. Dès lors que s'opère un déplacement lié à la sublimation, le brouillage des codes dans la représentation des genres suscite alors, dans sa valeur transgressive, une curiosité propre: enjeu du queer dans le cuir participant d'une déconstruction qui « provoque » au sens étymologique du mot, c'est-à-dire qui « invite à sortir » du cadre normé (Villemur 2007). Jusqu'à inverser les enjeux de profondeur et de surface. Ainsi l'œuvre de Jana Sterbak, de Vanitas, robe de chair pour albinos anorexique $^{16}$ (1987) à Distraction ${ }^{17}$ (1993-99), montre les dessous du corps en un prêt-àporté où l'intérieur et l'extérieur sont confondus : pour l'une, une robe de viande animale dont la chair est à la fois vêtement et peau, pour l'autre, une chemise transparente sur laquelle l'artiste a fixé un système pileux...

\section{Pilosité et transgression du nu}

4 On se souvient qu'en 1989, un groupe d'activistes féministes, les Guerrilla Girls, lançait le slogan « Do Women have to be naked to get into the Met Museum? Less $5 \%$ of the 
artists in the Modern Art sections are women, but $85 \%$ of the nudes are female ${ }^{18}$ ", et affublaient la Grande Odalisque d'Ingres d'une tête poilue de King Kong anonyme.

Une artiste contemporaine, vidéaste, Katarzyna Kozyra (née en 1963) parodiant le modèle de l'olympia ${ }^{19}$, a détourné ce canon du $\mathrm{Nu}$ par une beauté vénusienne ayant perdu ses cheveux. Si elle est chauve, ce n'est pas à cause d'un haut niveau de testostérone dans le sang, mais bien plutôt Kozyra élimine la chevelure comme dernier signe de vitalité associé à la féminité. Car dans ce qui est un autoportrait, le corps de la courtisane parodié est devenu corps malade, transformé par la chimiothérapie que subit alors l'artiste. L'étrangeté de la figure surgit dès lors au croisement de la mise en critique d'un modèle et d'un dérèglement de la nature. Du coup, Kozyra magnifie le corps mis à l'épreuve par la maladie comme elle détourne la nudité de tout voyeurisme. Avec Men's Bathhouse ${ }^{20}$ de 1999 (fig. 1), dans le contexte conservateur de la Pologne contemporaine, Kozyra prolonge son regard caustique sur la normalisation des comportements et des identités sexuelles, et sur la standardisation des corps. L'artiste ne pratique pas le cross-dressing pour revisiter des identités établies, mais travaille sur la comédie des apparences à partir de postiches (fausse pilosité du torse, fausse moustache, fausse barbe, faux poils pubiens et faux sexe), afin de s'introduire avec une caméra cachée, dans le milieu des bains publics réservé aux hommes. Présentée à la Biennale de Venise en 1999, l'installation retransmet la vidéo sur les doubles faces d'un pavillon octogonal tandis qu'un moniteur retrace à part les préparatifs de cette métamorphose. Introduit dans l'univers des bains, le spectateur est placé dans une position de voyeur alors même qu'il sait qu'elle n'est pas un homme, et que les hommes du bain l'ignorent. Cet écart permet de mesurer la méprise autant que l'injonction culturelle à laquelle sont tenus les corps sexués de se soumettre à des normes de comportement face à la trop évidente nudité. Si ce corps dans cet espace est perçu par le spectateur (qui a pris connaissance en amont du dispositif) comme celui d'un homme qui est une femme travestie de postiches, tandis que que l'artiste a caché ses seins sous une serviette et qu'elle dispose pour seul passeport de sa pilosité et de son pénis, les hommes au bain l'ignorent, qui ne lui prêtent pas attention sinon certains d'entre eux qui, par le jeu des regards et de l'attente, semblent volontiers la percevoir "gay", ainsi qu'elle se sent elle-même le devenir. D'une part, bien que se sachant déguisée, Kozyra s'est perçue dit-elle, "complètement nue ${ }^{21}$ " (Kozyra 77), c'est-à-dire sans protection, parce qu'elle avait intériorisé la nudité de son sexe et qu'elle se savait faussement couverte. D'autre part, «ce fut une révélation et un triomphe » remarque-t-elle, parce qu'il lui sembla «acquérir un genre" par-delà sa peur du sexe. "Personne ne m'a imaginé en tant que femme, mais les hommes m'ont perçue comme gay " et de poursuivre: «leur conviction que j'étais un gay me cacha mieux que n'importe quel travestissement " (Kozyra 76). Notons que Kozyra a également expérimenté en femme les bains publics de femmes, dans lesquels remarque-t-elle, les femmes ne s'observent pas (Kozyra 78). Ainsi le faire croire autant que l'ayant cru, pris au piège de la vraisemblance, creusent cette évidence d'une nudité toujours affolante et qu'il faut savoir recouvrir d'intention désirante ou d'inattention convenue. Car tout se passe comme si l'artiste découvrant qu'elle avait intériorisé la bicatégorisation sexuelle dans son travestissement, en appelait au genre pour sortir d'une peur dont on voit en fait que le genre lui-même repose sur une bicatégorisation. La performance de crossgendered identity expérimentée par Katarzyna Kozyra dans l'intrusion de lieux réservés, a révélé les rapports entre sexe, genre et sexualités, relation culturellement construite entre celui qui voit et celui qui est vu, selon que l'adresse vise un homme ou une 
femme, un genre ou un autre, une sexualité ou une autre (Dorlin). Le travail sur la pilosité, comme dispositif, sert d'interface à une (re)mise en jeu de ces catégories : ainsi le queer du poil défait-il la curiosité à l'endroit de l'autre sexe et de l'autre genre, pour ouvrir l'horizon d'attente d'une queeriosité qui dénouerait l'identification entre genre et sexe - une perspective de construction de l'identité queer jouant avec les prothèses et autres appendices.

Chez Daniela Comani (née en 1965) il ne s'agit pas non plus à strictement parler de travestissement dans son travail multi-média ${ }^{22}$. L'artiste déclare : «Je suis moi-même le sujet du travail, en un double autoportrait: dans le rôle du mari et de l'épouse, un heureux couple marié $\aleph^{23}$. Dans ce qui pourrait apparaître comme la banale reproduction de clichés masculins et féminins, Un matrimonio felice (2003-2006), photomontage numérique qui ne retouche ni les visages ni les accessoires, Daniela Comani tente avec des vêtements pris dans sa garde-robe, de donner existence à plusieurs identités sans réussir à les épuiser. La référence au livre de Judith Butler (Gender Trouble) qu'elle tient dans les mains, souligne ici la dimension queer et mélancolique qui participe à l'élaboration de genre. On sait avec Butler, que dans le processus d'acquisition de l'identité de genre, le moi s'approprie les attributs de l'autre à travers des " actes magiques d'imitation " (Butler 148). Ce qui est performable relève d'un processus par lequel une partie du pouvoir érogène du corps, abandonnée et devenue sexuellement imperformable, revient en surface travailler ce qui reste - par là, le corps est requalifié en son genre. Aucun érotisme, aucune félicité ne se dégage de ce matrimonio felice, si ce n'est l'entente neutralisante entre les deux personnages qui vont toujours par paire, pris à une inséparable polarité. A travers la distinction par la pilosité et par des conduites corporelles genrées, se rejoue une norme d'autant plus visible que ce couple repose sur une figure gémellaire doublement performée par l'artiste. En jouant perpétuellement le transfuge de genre avec elle-même, Daniela Comani joue moins sur les petits accommodements de la vie de couple que sur une différence infime disséminant des mises en situation du corps à la manière d'une bande de Moebius où l'endroit continue l'envers en une seule face mobile: si je bouge tu bouges, Je est un Autre, etc., que sa série des Double drawings (2000) sur papier transparent révèle autrement. "A double-gendered role-split self " ${ }^{24}$ où elle assume tous les rôles en une même identité. L'œuvre de Comani fait ainsi s'évaporer la différence biologique que l'on croit toujours première au profit d'une construction des genres relevant de modèles établis. Comani renvoie aussi l'hétérosexualité au vieux mythe platonicien de l'androgyne: unique et dédoublé dans l'un et l'autre de la différence, tel est ce matrimonio felice, qui fait d'un compromis un protocole ${ }^{25}$. Ainsi à travers ces visages gémellaires et pourtant différents, où la pilosité permet de conforter un genre dans un sexe, c'est finalement la similarité dans la différence que l'on repère et reconnaît.

7 En revanche, Del LaGrace Volcano qui qualifie son travail de «mutation délibérée ", expose des territoires méconnus ou volontairement ignorés, repoussant les limites du corps. Artiste londonien, Del se définit comme "a gender variant visual artist $^{26}$ ». Transexuel FTM (Female to Male), après avoir pris des hormones mâles, Del LaGrace Volcano qui est passé à une nouvelle vie dans laquelle il/elle se dit «queer identified intersexuel »- en 2005, confirme :

I access 'technologies of gender' in order to amplify rather than erase the hermaphroditic traces of my body. I name myself. A gender abolitionist. A part time 
gender terrorist. An intentional mutation and intersex by design (as opposed to diagnosis $)^{27}$.

8 La série des Gender Optional (2000) montre le photographe vivant tel un "living intersexed specimen ", muant plusieurs fois d'identité de genre. Del est à la fois F et M, ne rejetant ni l'un ni l'autre, jouant en alternance avec les genres, ouvrant sur une dimension hermaphrodite. Queer ou transgenre selon, Del donne à voir une multitude de possibles corporels, refusant le découpage binaire et exclusif des sexes comme des genres. Dans Debby's Muscles, Del est juste un peu trop butch pour être une femme, elle ferait plutôt bien une drag queen. De même, Delboy au crâne rasé est juste un peu trop vulnérable pour s'exposer en homme straight. La pilosité déplace ici les catégories de genre : cette soi-disant femme dévoile des jambes poilues et découvre des aisselles abondamment fournies, ce soi-disant homme cache sa pilosité sous une longue robe, tandis que des poils se voient sur son visage. En un double mouvement inverse, du dévoilement du bas et du haut du corps, les deux Volcano où se laisse voir Grace, et vice-versa, ne sont que les deux faces d'un même individu. "Ph/autographe» (Del LaGrace Volcano 6-11), l'heureuse expression de Jay Prosser à son endroit, appelle bien ce travail photographique en autobiographie. Des Transportraits aux Transgenital Lanscapes, Del LaGrace Volcano travaille le corps transsexuel en tant que corps mutant : la série des Mutating Self Portraits (2000) avec Del, Del Skin, Daddy Del et Andro Del, dont les titres mettent en évidence l'article défini del comme attache d'un indéterminé qui pourrait servir de copule entre LaGrace et Volcano, est une série qualifiée de Gender Optional, qui joue sur le déplacement de la pilosité, d'un plus vers un moins, dans laquelle le rasage intégral de l'androgynie affirme la neutralisation des genres dans la figure parfaitement lisse d'Andro Del (Del LaGrace Volcano 176). Dans la gradation de la pilosité, le travail de déplacement est ici horripilant, à savoir lié au frisson, au sens même de ce qui est hérissant pour le poil, et qui fait sensation et sens (le terme étant dérivé d'« horripilateur » qui se dit du muscle relevant chaque poil). Cette fluctuation de la pilosité à fleur de peau fait surgir une certaine transgression du beau, une mise en péril de la forme attendue et convenue. Se dessaisissant de tout repère après l'avoir travaillé, Volcano suscite moins un trouble des genres dans le jeu concerté d'une certaine ambiguïté, qu'il ne soulève l'inconvenance comme ultime, dans une ambivalence délibérément choisie, il travaille l'incongruité avec humour. L'artiste met le beau en péril, le terrible surgit au cœur de l'apparition du «trans ». En déstabilisant l'identité de sexe et de genre, l'approche queer de Volcano travaille le processus de sublimation jusqu'au point où "déconstruire " revient à affirmer un nouveau "constructivisme", fondé sur l'émergence du corps transgenre. Le travail de Del LaGrace Volcano questionne la masculinité lesbienne, complique la lecture de la féminité, ouvre celle du transgenre et de l'intersexe, célèbre les corps des butch dykes comme des hermaphrodykes, vise dans une saisie exponentielle des différences transgenres à de sublime mutations - titre de son livre. Le terme de sublime souligne ici combien le détournement des forces pulsionnelles vers de nouveaux buts est un déplacement producteur de valeurs de genre. Loin d'évoquer la sublimation comme effet du sublime, ou de traiter du sublime comme déflagration de la frontière entre le moi et l'autre ${ }^{28}$, Del LaGrace Volcano expose dans un certain dépassement de soi, une mutation qui atteint à une nouvelle dignité, en une catharsis à la fois médicale et esthétique, dont les "transgenital landscapes» se veulent l'aboutissement Hermaphrodite Torso (1999), Lazlo and Shanti (2004). Le transgenre, le queer et l'intersexe ainsi vont jusqu'à se croiser chez Del LaGrace Volcano dans une forte affirmation 
identitaire. Si le «queer est partout et nulle part » aux dires de François Cusset, nous ne le suivrons pas ici quand il affirme que le "queer est là où je ne suis pas, là où le je n'est pas » (Cusset 8) : de fait chez Del LaGrace Volcano l'approche queer du « je ne suis pas là » n'infirme pas que le je n'y est pas. L'épuisement des potentialités du moi n'aboutit pas à l'évidement du moi, à une disparition du sujet, mais bien au contraire conduit à l'affirmation aporétique d'un corps qui se veut à sa manière glorieux. Opérant une mutation dans l'ordre de l'organique, œuvrant avec la plasticité et la singularité des corps, Del LaGrace Volcano montre par là combien le nu est mise en mouvement de l'identité, corps sublimé à travers des catégories de genres, autant qu'il fait preuve dans son travail sur l'intersexualité d'une critique du processus de sexuation progressive des corps. Si les photographies offrent l'impossibilité d'une identification précise de la sexualité du modèle, la nudité est comme bouleversée par la volonté d'amplifier plus que d'effacer les traces genrées ou intersexuées du corps. Ainsi, se refusant à être enfermé dans la bi-catégorisation des êtres humains, comme dans la rhétorique binaire du cross- et du trans-, Volcano démontre que le sexe « biologique » n'est pas réductible à la reproduction sexuée, pas plus que les genres ne le sont aux caractères dits secondaires ${ }^{29}$. Ainsi Del LaGrace Volcano dans une approche queer et abolitionniste du genre, affirmant la nature corrosive et transitoire du queer (Villemur 2015), vise des stratégies de réappropriation et de resignification résolument liées à une logique postidentitaire.

9 La migration de la pilosité révèlerait donc ce transit infini des identités jusqu'au transgenre anti-essentialiste et anti-transsexuel ${ }^{30}$. Et l'on peut se demander si le poil a un genre, ou s'il fait toujours genre. "La sublimation, écrit Norman Brown, est une compensation alloplastique de la perte de Soi $»^{31}$ (Brown 133-134). La fonction de la tonsure dans son rapport au sacré, l'acte quotidien de se raser dans son contrôle sur la mort participent toutes deux d'une renaissance symbolique. Maîtriser le poil ou se l'approprier en un geste apotropaïque pour en saisir la puissance magique, c'est se rendre maitre de la différenciation de sexe et de genre telle qu'elle s'est construite. Au tout début de sa carrière, Ana Mendieta (1948-1985) engage sa pratique de la performance dans une double stratégie, à la fois sur le mode opératoire magique d'appropriation des attributs de l'autre, et sur le mode du détournement des canons esthétiques, ceux du glabre et du lisse qui entrent dans la définition du Beau dans l'art. À partir des années soixante-dix, les drag performances d'un certain nombre de ses contemporaines, celles de Eleanor Antin (The King, 1973), de Martha Wilson, d'Adrian Piper, interrogent, toutes diversement, les masculinités. Inversement les gender-cross performances, ainsi de celle de Vito Acconci (Conversions, 1971) où ce dernier éradique les poils de son torse, pétrit ses pectoraux pour faire apparaître des seins, cachant son pénis entre ses jambes, conduisent à une appropriation et à une réélaboration du féminin à partir d'un corps masculin. En 1972, dans une première performance réalisée dans le cadre d'un travail à l'université de l'Iowa ${ }^{32}$, Ana Mendieta colle sur son visage la barbe du poète Morty Sklar qui en découpe des bouts devant un miroir. Des photographies dignes de l'anthropométrie immortalisent son visage barbu de face et de profil, précédées par celle du visage de Sklar avant l'intervention; ainsi qu'une photographie de tous deux, l'un au-dessus de l'autre, lui avec sa seule moustache, elle avec une barbe fournie. Dans une deuxième performance, elle ne colle que sa moustache et fait pareillement apparaître le résultat final. Qu'est-ce qui est en jeu dans cet échange de Facial Hair Transplant? Ana Mendieta affirme à propos de ce travail qu'elle avait toujours été fascinée par la pilosité quelle qu'elle soit, la chevelure de 
Samson, celle des scalps pratiqués par les Cheyennes, la moustache ajoutée à la Joconde par Duchamp dont elle retient les propos (Duchamp voyait la Joconde alors transformée en homme et non pas telle une femme déguisée en homme). C'est même au regard de $L$. H. O. O.Q. de 1919, que Mendieta a placé son travail comme une extension de l'œuvre de Duchamp. Elle retient donc que Duchamp s'était étonné de voir Mona Lisa transformée en homme, une fois affublée d'une moustache et d'une barbichette (Breitwieser 216-228). Est-ce qu'elle expérimente la même chose ? L'anthropologie nous a appris à voir dans l'effet d'appropriation de ces attributs un effet de captation de l'autre ${ }^{33}$, tant afin de neutraliser le danger que représente toute altérité que pour en récupérer la force vive. Avec Facial Hair Transplant (1972), l'idée de Mendieta est de transférer d'une place à une autre, sur son visage, la barbe et la moustache de son ami : elle pense que transférer ainsi la pilosité d'une personne à une autre, c'est opérer un déplacement de puissance. Mendieta est alors à la recherche de la puissance des images dans leur sens magique $^{34}$. Une décennie plus tard, elle avoue que son « art a grandi sur la croyance en une énergie universelle qui court à travers toute chose » (Breitwieser 228), un fluide qui circule dans le macrocosme et qu'il faut savoir capter pour lui donner forme. Mendieta déplace la citation de Duchamp en un travail d'appropriation par lequel elle juge que le résultat final non plus, ne ressemble pas à un « déguisement » : elle a vécu l'expérience comme si cette barbe était devenue sienne $\mathrm{e}^{35}$. Ainsi par un art corporel où elle se met volontiers en jeu, Ana Mendieta se démarque de l'art conceptuel qui domine son époque. Il faut rappeler que ce travail précède la série des Blood Writing, Blood Sign et Body Tracks de 1973-74, où l'artiste s'affirme dans le body art en utilisant son propre sang ainsi que dans un rituel magique ${ }^{36}$. Dans Glass on Body (1972), elle pratique la défiguration de soi par l'écrasement de son visage sur une plaque de verre qui aplatit les masses molles du visage, créant ainsi de nouveaux plis et contours. Dans Facial Cosmetic Variations (1972-1974), le travail de déformation du visage passe de l'état lisse créé par l'enveloppe d'un bas enfilé effaçant tout le volume de la chevelure, à des cheveux dont la masse travaillée par la mousse d'un shampoing et ramenée par mèches par-devant, sur le menton et noués sous le nez, l'est à la manière d'une barbe ou d'une moustache. CEuvrant volontiers sur la confusion entre cheveux et système pileux, Ana Mendieta avec Facial Hair Transplant (1972) s'écarte de la première Mona Lisa en ajoutant une pilosité (on sait que Léonard avait dépeint épilés les sourcils de La Joconde) et déplace le travail de Duchamp sur l'inframince (ce concept de la différence au sein d'une ressemblance négative ${ }^{37}$ ) Le procédé de citation reconduit la question de l'inversion du genre que posait déjà Duchamp, pas seulement entre le masculin et le féminin mais aussi entre le visuel et le tactile. Chez Duchamp, la notion d'inframince est en relation avec la ressemblance, c'est une distinction minime pour ne pas dire infime, une "séparation qui a les deux sens mâle et femelle » (Duchamp 22). «Le détail pileux privilégie l'ambiguïté sexuelle. [...] Il semble le moyen et l'indice de l'inversion des genres " (Badet 20). La chair pubescente relève de l'inframince comme le corps rasé : le poil est un séparateur pour Marcel Duchamp, une différence séparative inframince entre des êtres identiques. Question de contact et d'affleurement. À partir de l'inframince, cette patine des choses longtemps caressées, et de l'inframince du poil qui impose à l'image du féminin sa valeur de négativité, une même angoisse surgit chez Duchamp. On connait sa phobie pour les poils ${ }^{38}:$ L. H. O. O. Q. est alternativement poilue en 1919, et rasée en 1965. En 1946, il glisse dans l'exemplaire de la Boite-en-valise destinée à Matta un portrait inframince simplement constitué de quelques touffes: cheveux, poils d'aisselles et poils pubiens scotchés au revers d'un plexiglas transparent. 
Le mannequin de Etant donnés: 1) La Chute d'eau, 2) Le Gaz d'éclairage (1946-1966) est totalement épilé (absence de toison pubienne, de pilosité sous les bras), et tactilement solarisé. With my tongue in my cheek (1959) est révélateur de cette hantise du poil, mais souligne aussi combien le poil fait signe en tant qu'inframince, dans ces replis optiquement surlignés et tactilement rentrants et saillants. With my tongue in my cheek, version dialectisée de la ressemblance optique et tactile, reformule la problématique du contour d'ombre déchiré de l'autoportrait de profil. La pilosité révèle ici un malaise dans la représentation : ce qui doit être vu de profil comme l'extérieur du visage surgit en fait de son support tel une chose frontalement exposée. La pilosité met en place un regard du toucher. Le poil n'a pas de genre pour Duchamp, inframince, il relève d'une mystérieuse différence séparative. En prenant l'histoire de l'art «à rebrousse-poil » dans son travail sur la Joconde, Duchamp utilise la pilosité comme pratique d'un écart, sur le mode opératoire de l'inversion. En revanche, Ana Mendieta joue sur du réversible alors même qu'elle tente de combler l'intolérable distance entre les êtres dans un rituel magique d'incorporation. À la différence des femmes moustachues et barbues de Jeanne Dunning ou de Zoe Leonard, Facial Hair Transplant traduit la dissymétrie qui existe dans l'échange. Le visage qu'elle tend à l'autre est le lieu d'une contamination du féminin par le masculin, sans que le féminin y perde sa part: il y a là comme l'effet d'un redoublement des polarités binaires. Moins que d'ambiguïté, il s'agit bien d'ambivalence dans la réversibilité des genres. Ana est visible sous la pilosité de Morty. Elle se charge de la différence de l'autre dans un autoportrait qui appelle l'altérité. D'une certaine manière, on est dans un processus inverse à celui de Frida Kahlo, qui dans son Auto-portrait aux cheveux coupés (1940) avait ajouté : « Vois, si je t'aimais, c'était à cause de tes cheveux; maintenant que tu as les cheveux coupés, je ne t'aime plus " ${ }^{39}$, œuvre réalisée dans un moment d'affranchissement, et d'indépendance par rapport à Diego Rivera.

10 Par contre, la dimension parodique chez Cindy Sherman développe des fictions identitaires qui déstabilisent les genres non par ce jeu d'ambivalence propre à Mendieta, mais par celui d'une ambiguïté qui traverse la dissemblance propre au régime des images (Villemur 2006). Ambiguïté qui gagne en force d'autant que le simulacre met en oubli l'original : Sherman n'est nulle part et partout, elle n'est jamais reconnaissable sous ses travestissements, et il ne s'agit pas d'autoportraits. Si elle démultiplie les avatars dans la déconstruction de la mascarade du féminin, il est remarquable qu'il lui a été plus facile d'aborder dans les History Portraits (1989) que dans les Film Stills (1977-80) la question de la représentation du masculin, au point de mieux jouer avec le masculin dans des attributs pour lesquels la distance historique était plus forte. «Les hommes d'alors ne paraissent pas plus éloignés de nous que ne le sont les femmes, dit-elle » (Francblin 18). La perfection de l'imitation est alors confondante parmi les hommes représentés de la série (Untitled \#194, 1989, et Untitled \#203, 1989 ou encore Untitled \#213, 1989 et Untitled \#218, 1990). Ironie du portrait : l'œuvre originale (ce qui fait tableau) est ici perdue cependant que le personnage historique semble bien vivant, à la manière d'un revenant, il hante notre regard à mesure que nous interrogeons l'improbable sexe féminin qui se cache derrière cette prouesse transformiste en l'autre sexe. La pilosité y est particulièrement marquée, jamais les hommes ne semblent avoir été aussi vrais chez Cindy Sherman. De fait, l'œuvre de Sherman peut se comprendre comme une entreprise apotropaïque visant à déjouer la fascination, le fascinum où se joue la construction de la différence des sexes, et donc à démultiplier l'offre visuelle, l'ostentation et l'exhibition. Mais c'est dans la mesure où 
l'original est perdu, que Cindy Sherman est partout et nulle part, et sûrement pas ellemême dans ces photos, que la porosité et le transfert de pilosité opèrent au mieux. Nous sommes bien loin du phénomène d'attraction et de répulsion que suscite la pilosité, tel qu'ont pu le traiter des artistes comme Jeanne Dunning ${ }^{40}$, Zoe Leonard ${ }^{41}$, ou encore Jeanne Mordoj qui a pu faire l'éloge du poil au théâtre ${ }^{42}$. Là, le thème de la femme à barbe y tient encore son rôle de merveille et de monstre détournant les critères esthétiques - il faudrait d'ailleurs rappeler ici le travail de réappropriation et de réhabilitation réalisé par Pauline Boudry et Renate Lorenz dans N.O. Body (2008), à partir des images d'Annie Jones, célèbre femme à barbe du Barnum Circus, dans une œuvre qui donne l'expression aux anormaux de Magnus Hirschfeld (théoricien de la transition sexuelle au début $d u \mathrm{XX}^{\mathrm{e}}$ siècle), en tant que sujets et non plus curiosité observée ${ }^{43}$.

Si la pilosité révèle une nudité coupable, au point d'ouvrir la possibilité d'une nudité coupée dans le rituel de la tonsure ou de l'épilation qui débarrasse le corps de son impureté, le geste créateur peut à son tour le travailler pour venir transgresser les normes esthétiques. Inventer de nouveaux corps en puissance, qu'il s'agisse de s'approprier l'autre par incorporation, de dévoiler une nature hybride ou transitoire, de semer le doute et de faire circuler autrement le désir de voir et de toucher : jusqu'à faire advenir une polymorphie du corps par-delà la simple inversion des genres. Comme si le queer était au poil ce que serait le trans à la peau: une question de profondeur à la surface.

\section{KATARZYNA KOZYRA (1963 - ), CAPTURE D'ÉCRAN DE MEN'S BATHHOUSE}

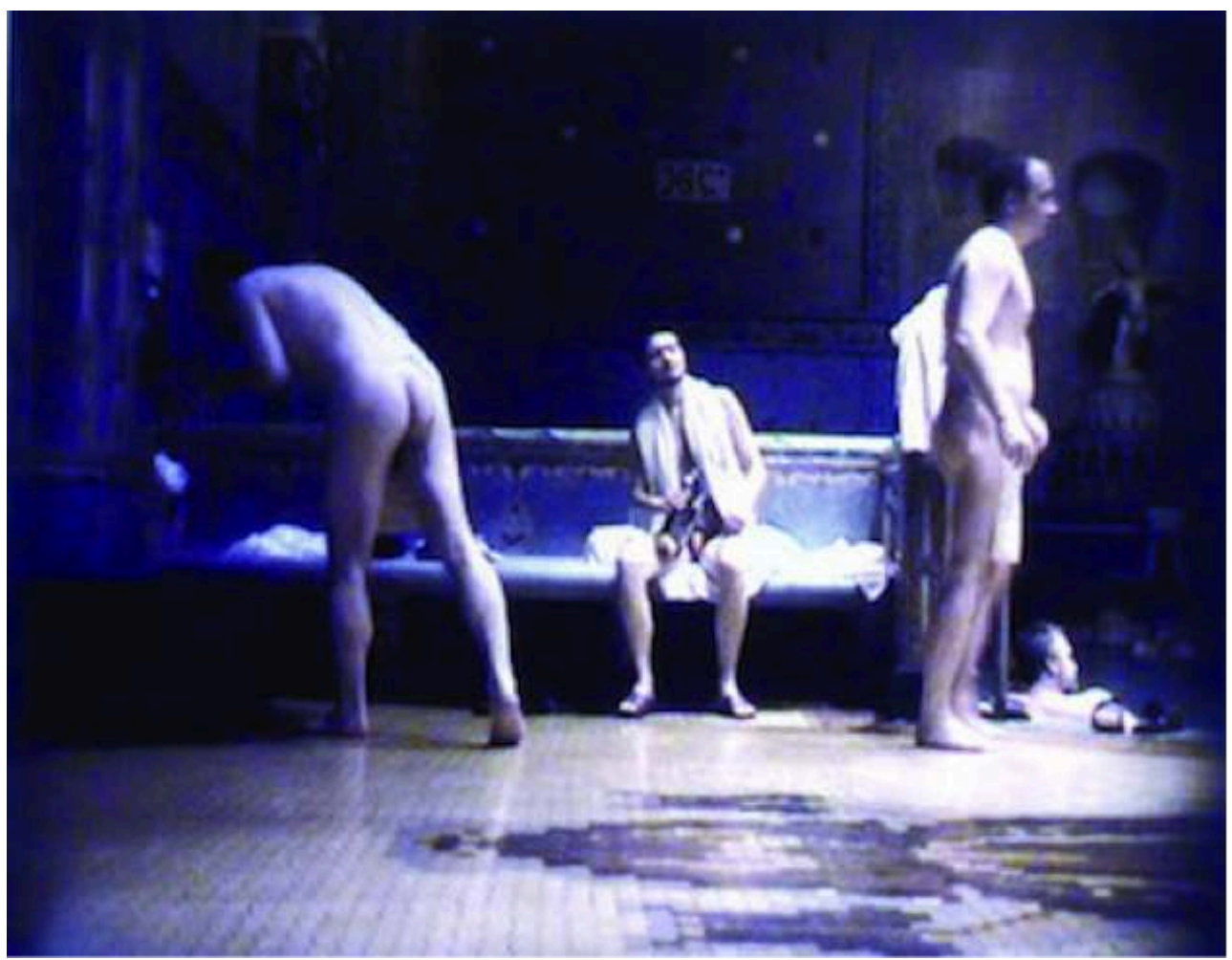

Installation vidéo, 5 canaux, sync. Loop, 4:3 PAL (1999. Biennale de Venise). 


\section{BIBLIOGRAPHIE}

Agamben, Giorgio. Nudités. Trad. Martin Rueff. Paris : Éditions Payot \& Rivages, 2009.

Auzépy, Marie-France, Cornette, Joël. Histoire du poil. Paris : Belin, 2011.

Badet, Muriel. « Petits exercices d'inversion des genres, des poils de L. H. O. O. Q. de Marcel Duchamp à la barbe d'Ana Mendieta.» Ligeia : 69-72 (2006) : 16-24.

Barreras Del Rio, Petra. Ana Mendieta, a retrospective, November 20, 1987-January 24, 1988. New York : The New Museum of Contemporary Art, 1987.

Bernadac, Marie-Laure, Marcadé, Bernard. Fémininmasculin. Le sexe de l'art. 24 octobre 1995-12 février 1996. Paris : Gallimard/Electa/Editions du Centre Pompidou, 1995.

Bereni, Laure, Chauvin, Sébastien, Jaunait, Alexandre, Revillard, Anne. Introduction aux études de genre. $2^{\mathrm{e}}$ édition revue et augmentée. Bruxelles : De Boeck Supérieur, 2012.

Bologne, Jean-Claude. Histoire de la pudeur. Paris: Olivier Orban, 1986.

Breitwieser, Sabine. Vivências/Lebenserfahrung/Life experience, Generali Fondation, 15 September bis 22-December 2000. Wien.

Butler, Judith. Trouble dans le genre. Pour un féminisme de la subversion. Trad. de l'américain par Cynthia Kraus. Paris : La Découverte, 2005.

Brown, Norman O. Life against Death: the psychoanalytical meaning of history. London : Routledge, 1959.

Clark, Kenneth. The Nude. A Study of Ideal Art (1956), Le Nu, trad. M. Laroche. Paris : Le Livre de Poche, 1969.

Cusset, François. « Intérieur queer. Plaisir sans corps, politique sans sujet.» Rue Descartes 40 (2003) 8-17.

De LaGrace Volcano. Sublime Mutations. Tübingen : Konkursbuch Verlag Claudia Gehrke, 2000. Didi-Huberman, Georges. Ouvrir Vénus. Nudité, rêve, cruauté. Paris : Gallimard, 1999.

Dorlin, Elsa. « Pour une épistémologie historique du sexe.» Araben 3 (2006): 8-19.

---. Sexe, genre et sexualités. Paris: PUF, 2008.

Douglas, Mary. De la souillure. Paris : Maspero, 1971.

Duchamp, Marcel. Notes. Paris : Flammarion, 1999.

Eribon, Didier (dir.). Dictionnaire des cultures gays et lesbiennes. Paris : Larousse, 2003.

Fausto-Sterling, Anne. Sexing the Body. New York : Basic Books, 2000.

Ferrari, Federico, Nancy, Jean-Luc. Nus sommes. La peau des images. Bruxelles : Yves Gevaert Editeur, 2002.

Fischer-Sarazin-Levassor, Lydie. Un échec matrimonial. Dijon, Les Presses du Réel, 2004. Francblin, Catherine. « Cindy Sherman, personnage très ordinaire.» Art Press janvier (1992).

Freud, Sigmund. Inhibition, symptôme et angoisse (1926), trad. M. Tort. Paris : PUF, 1978.

---. Trois Essais sur la théorie sexuelle. Paris : Gallimard, 1987. 
Gagnebin, Murielle, Milly Julien (dirs.). Les images honteuses. Paris : Champ Vallon, 2006.

Josse, Béatrice. « "Deux ou trois choses que j'ignore d'elles". Pour un manifeste post( ?)-féministe. » Metz: Frac de Lorraine, 2008.

Kozyra, Katarzyna. The Men's Bathhouse. Galeria Zacheta, Warsaw, XLVIII International Biennal of the Visual Arts, Venice : Polish Pavilion, 1999.

Loviot, Louis. Alice Ozy. Paris : Les Bibliophiles fantaisistes, 1910.

Maertens, Jean-Thierry. Le corps sectionné. Ritologiques 2. Paris : Aubier-Montaigne, 1978.

Mulvey, Laura. « Fantasmagorie du corps féminin » in Cindy Sherman. Paris : Flammarion-Jeu de Paume, 2006. 284-303.

Prosser, Jay. « The art of Ph/Autography : Del LaGrace Volcano. » In Sublime Mutations. Volcano De LaGrace. Tübingen: Konkursbuch Verlag Claudia Gehrke, 2000. 7-11.

Quillet, Lucile. «Filles poilues, garçons épilés : l'expérience insolite d'étudiants américains.» Le Figaro Madame 9 juillet (2014).

Rey, Alain. Dictionnaire historique de la langue française. Paris : Le Robert, 1992.

Saint Girons, Baldine. Le Sublime de l'Antiquité à nos jours. Paris : Desjonquères, 2005.

Valéry, Paul. L’Idée fixe. Cuvres complètes. Paris: Gallimard, coll. « La Pléiade », 1960.

Vaton, Marie, « La revanche des poilues », Le Nouvel Observateur 2215 (2007)

Vergez-Seija Sarah (dir.). La Peau. Un continent à explorer. Paris : Autrement, 2005.

Villemur, Frédérique. Figures de l'androgyne, doctorat d'Histoire, sous la direction de Michelle Perrot, Paris 7, 1991.

---. « Le dissolvant du queer est-il soluble dans l'air? » in Esthétique(s) queer dans la littérature et les arts. Sexualités et politiques du trouble. Ed. Muriel Plana, Frédéric Sounac. Dijon: Editions Universitaires de Dijon, 2015.

---. « Le Neutre et l'Inframince : Duchamp et la mise à nu », in Annette Runte (dir.). Machine littéraire, machine célibataire et 'genre neutre'. Würzburg : Königshausen \& Neumann, 2011. 237-253

---. « Pensée queer et mélancolie du genre. » Cahiers du Genre 43 (2007) : 149-165.

---. « Trouble de genres en vue : Cindy Sherman.», in Création au féminin, vol. 2 : Arts visuels. Ed. Marianne Camus. Dijon : Editions Universitaires de Dijon, 2006. 57-67.

Wagner, Frank, König, Kasper, Friedrich, Julia. Das achte Feld : Geschlechter, Leben und Begehren in der Kunst seit 1960/The Eighth Square : Gender, Life, and Desire in the Arts since 1960, 19 August-12 November 2006, Köln, Museum Ludwig. Köln : Hatje Cantz Verlag, Ostfildern, 2006.

\section{NOTES}

1. Le $\mathrm{Nu}$, invention formelle (art form) par opposition à la nudité (nakedness), est une catégorie mise en place dans le discours de l'histoire de l'art par Kenneth Clark dans son ouvrage The Nude. A Study od Ideal Art (1956). Pour une critique, se reporter à Georges Didi-Huberman, Ouvrir Vénus. Nudité, rêve, cruauté.

2. «Ce qu'il y a de plus profond en l'homme, c'est la peau... En tant qu'il se connaît » (Valéry 217).

3. Article "Queer» par Xavier Lemoine, qui souligne cette remarque étymologique d'Eve Sedgwick (Eribon 395). 
4. Voir Schulié, Robin. « Systèmes pileux.» (Vergez-Seija 26-41)

5. Voir «Filles poilues, garçons épilés : l'expérience insolite d'étudiants américains » (Quillet) pour le Figaro Madame, 9 juillet 2014.

6. Jan Van Eyck, Retable de l'Agneau mystique: Adam et Eve, 1426-1432, huile sur bois. Gand, cathédrale Saint-Bavon.

7. Lors de son passage à Gand en 1781, Joseph II s'indigne de la nudité d'Adam et Ève et exige que les panneaux soient démontés. À la fin du $19^{\text {ème }}$ siècle, les pièces exposées sont des copies dont les figures sont habillées, la nudité d'Adam et Ève étant jugée choquante dans une cathédrale, c'est alors que les deux panneaux originaux sont vendus à l'État belge en 1861 et déposés aux Musées royaux des beaux-arts de Belgique à Bruxelles.

8. Giorgione et Titien, Vénus endormie, vers 1509, huile sur toile. Dresde, Gemäldegalerie.

9. Sur cette question voir : Didi-Huberman et Agamben.

10. Rubens, Hercule et Omphale, 1602-1605, huile sur toile, Paris, musée du Louvre.

11. Edouard Manet, Olympia, 1863, huile sur toile. Paris, musée d'Orsay.

12. Gustave Courbet, Femme nue couchée, 1862, huile sur toile, collection privée. Toile redécouverte après la deuxième guerre mondiale et récemment exposée au Grand Palais à Paris, Gustave Courbet, 13 octobre 2007-28 janvier 2008.

13. Théodore Chassériau, Baigneuse endormie près d'une source, 1850, huile sur toile. Avignon, musée Calvet.

14. Révélateur est à ce titre le poème de Théophile Gautier à ce sujet : «Pentélique, Paros, marbres neigeux de Grèce / Dont Praxitèle a fait la chair de ses Vénus / Vos blancheurs suffisaient à ces corps de déesses... Noircissez, car Alice a montré ses seins nus.» (Loviot 24).

15. Alexandre Cabanel, La naissance de Vénus, 1863, huile sur toile. Paris, musée d'Orsay.

16. Voir l'œuvre sur : https://www.centrepompidou.fr/cpv/resource/cjyy4gX/ryjdGjo

17. Voir l'œuvre sur : http://www.artnet.fr/search/artworks/?q=sterbak\%20distraction

18. «Faut-il que les femmes soient nues pour entrer au Metropolitan Museum ? Moins de $5 \%$ des artistes de la section d'art moderne sont des femmes, mais $85 \%$ des nus sont féminins ».

19. Katarzyna Kozyra, Installation, 1996, l'œuvre comprend trois grandes photographies et une vidéo de $12 \mathrm{~min}$.

20. Voir des extraits de l'installation et de la vidéo, mise en ligne le 24 avril 2012 : https:// www.youtube.com/watch?v=_cRIcbb9D2o

21. Artur Zmijewski interviewe Katarzyna Kozyra, «A Passport into the Male Sanctum».

22. Se reporter à son site pour ses œuvres : www.danielacomani.net

23. Je traduis de l'italien. Pour Un matrimonio felice se reporter à cette série d'une grande qualité d'exposition : http://www.danielacomani.net/ehe03_12.html

24. http://www.danielacomani.net/works.07.html. Un matrimonio felice, A Happy Marriage : introduction.

25. Sur la notion de « compromis androgyne » je me permets de renvoyer à mes travaux de thèse (Villemur 1991).

26. Voir son site : www.dellagracevolcano.com.

27. http://www.dellagracevolcano.com/statement.html

28. Sur cette question, je renvoie à Baldine Saint Girons.

29. Sur ce point se reporter à Anne Fausto-Sterling et Elsa Dorlin.

30. Se reporter à Laure Bereni, Sébastien Chauvin, Alexandre Jaunait, Anne Revillard dans leur ouvrage Introduction aux études de genre, sur les notions de transsexualisme, transgendérisme, et cisgenre.

31. Je traduis de l'anglais.

32. Cette performance s'inscrit pour l'obtention d'un Master of Arts pour The School of Art and Art History in the Graduate College of The University of Iowa. 
33. Voir Jean-Thierry Maertens.

34. "My paintings were not real enough for what I wanted the images to convey, and by real I mean I wanted my images have power, to be magic.» (Barreras Del Rio 28).

35. « After looking at myself in a mirror, the beard became real. It did not look like a disguise. It became part of myself and not at all unatural to my appearance.» (Breitwieser 216).

36. "I started immediately using blood, I guess because I think it's a very powerful magical thing. I don't see it as a negative force.» (Barreras del Rio 29).

37. Sur la notion d'inframince ici à l'œuvre : Duchamp (19-47) et Villemur (2011).

38. Marcel Duchamp n'aimait pas les poils, ainsi qu'en témoignent Lydie Fischer-SarazinLevassor dans son ouvrage Un échec matrimonial et son œuvre finale, Étant donnés : $1^{\circ}$ ) la chute d'eau, $2^{\circ}$ ) le gaz d'éclairage (1966).

39. Frida Kahlo, Auto-portrait aux cheveux coupés, 1940, huile sur toile. New York, The Museum of Modern Art.

40. Jeanne Dunning, Sans titre, 1988, photographies, FRAC Poitou-Charentes.

41. Ainsi en 1997, pour un Bearded Lady Calendar, Zoe Leonard propose à son amie barbue Jennifer Miller de prendre une pose de pin-up à la Marylin Monroe.

42. Jeanne Mordoj, Éloge du poil, Cie Bal, 2007. Circassienne ventriloque, Mordoj joue sur un malaise d'identification sur fond d'empathie.

43. Pauline Boudry et Renate Lorenz, N. O. Body, installation avec film en $16 \mathrm{~mm}$ et 47 photographies, $15 \mathrm{mn}, 2008$. «La question que pose le film N.O. Body est la suivante : que résultet-il du lien entre la normalité et la déviance quand l'objet étudié se met dans la peau du scientifique et, se mettant à rire, fait se redérouler l'histoire, à partir de sa position inversée? ". Voir : http://www.boudry-lorenz.de/n-o-body/

\section{RÉSUMÉS}

Confronter dans l'art contemporain les genres masculin et féminin, dont on sait qu'ils sont culturellement construits, à travers la notion même d'inversion dans une approche queer du détournement, a permis d'ouvrir un champ d'investigation sur la valeur transgressive ou non de leurs usages, et de confirmer ce que le trouble dans le jeu « du même et de l'autre » venait à déstabiliser, pour laisser ouverte la question de l'identité par-delà ces catégories binaires. On interroge ici le transgenre et le corps sublimé autour de la question queer de l'inversion des genres quand le poil sollicite diversement la curiosité dans l'acte esthétique. Le fait de se travestir en se collant des poils (tout en laissant transparaître ou non une identité antérieure) ou le fait de s'engager dans des mutations transgenres (en une catharsis à la fois médicale et esthétique, questionnant tout autrement l'exhibition des poils), présente un brouillage des codes jouant sur des écarts dont on visera à dégager la valeur transgressive autour des œuvres de Del LaGrace Volcano, Daniela Comani, Katarzyna Kozyra, Ana Mendieta et Cindy Sherman. La métamorphose des corps, en tant que "changement au-delà ", sera donc posée en surface, à partir de l'inframince du poil et de l'identité de genre.

Confronting male and female genders in contemporary art through the notion of reversal in a queer approach, has opened a field of investigation on their possible transgressive value, and confirmed the destabilization that the interplay between sameness and otherness operates, leaving open the question of identity beyond binary categories. We question transgender identity 
and the sublimated body around the queer issue of gender reversal and the role played by the hair in arousing aesthetic curiosity. To cross-dress by sticking hairs on one's body (while not concealing the previous identity) or to engage in transgender mutations (in both a medical and aesthetic catharsis which questions the exhibition of hairs), blurs the codes and plays on the differences whose transgressive value we will try to identify in the works of Del LaGrace Volcano, Daniela Comani, Katarzyna Kozyra, Ana Mendieta and Cindy Sherman.

\section{INDEX}

Mots-clés : poil, nu, nudité, travestissement, body art, queer, transgenre

Keywords : hair, nude, nudity, crossdressing, body art, queer, transgender

\section{AUTEURS}

\section{FRÉDÉRIQUE VILLEMUR}

Maître de conférences HDR en histoire de l'art

École nationale supérieure d'architecture, Montpellier.

fr.villemur@gmail.com 